\title{
Multi-Class Energy Management for Peer-to-Peer Energy Trading Driven by Prosumer Preferences
}

\author{
Thomas Morstyn, Member, IEEE, and Malcolm D. McCulloch, Senior Member, IEEE
}

\begin{abstract}
This paper proposes a peer-to-peer energy market platform based on the new concept of multi-class energy management, to coordinate trading between prosumers with heterogeneous (i.e. beyond purely financial) preferences. Power networks are undergoing a fundamental transition, with traditionally passive distribution network consumers becoming 'prosumers'; proactive consumers that actively manage their production and consumption of energy. The paper introduces the new concept of energy classes, allowing energy to be treated as a heterogeneous product, based on attributes of its source which are perceived by prosumers to have value. Examples include generation technology, location in the network and owner's reputation. The proposed peer-to-peer energy market platform coordinates trading between subscribed prosumers and the wholesale electricity market, to minimise costs associated with losses and battery depreciation, while providing added value by accounting for the prosumers' individual preferences for the source/destination of the energy they consume/produce. The decomposable structure of the multi-class energy management problem is exploited to devise a distributed price-directed optimisation mechanism, providing scalability and prosumer data privacy. Receding horizon model predictive control allows the prosumers to adjust their planned power flows based on the wholesale energy price, and up-to-date renewable generation and load predictions.
\end{abstract}

Index Terms-Distributed Optimisation, Dynamic Optimal Power Flow, Energy Management System, Energy Class, Energy Market, Microgrid, Peer-to-Peer, Prosumer

\section{INTRODUCTION}

$\mathbf{P}$ OWER networks are undergoing a fundamental transition, with traditionally passive distribution network consumers becoming 'prosumers'; proactive consumers, with local generation sources and/or energy storage systems, that actively manage their consumption and production of energy [1].

Previously, limited communications and control infrastructure at the distribution level has not allowed small consumers to directly interact with the wholesale electricity market. Instead, they have signed a contract with a retail supplier, and the supplier buys energy from the wholesale market on their behalf [2]. Under these contracts, the consumers are individually metered so that they can be charged for energy used, and paid a feed-in tariff for energy supplied to the network.

Under a contract based on individually metered usage, prosumers with energy storage capacity only benefit from shifting their locally generated energy [3]. However, this neglects the

This work is supported by the Engineering and Physical Sciences Research Council (EP/N03466X/1) and the Oxford Martin Programme on Integrating Renewable Energy

T. Morstyn and M. D. McCulloch are with the Department of Engineering Science at the University of Oxford, Oxford OX1 2JD, United Kingdom (email: thomas.morstyn@eng.ox.ac.uk, malcolm.mcculloch@eng.ox.ac.uk). additional value that coordinated control of distributed energy storage systems can provide by reducing losses and congestion [4]. In addition, coordinating local energy storage systems based on the wholesale energy market allows them to respond to upstream energy needs [5].

The problem of coordinating bidirectional power flows between distribution network prosumers has been studied as the optimal energy management problem, i.e. the problem of scheduling the power flows between energy storage systems over a time horizon, considering renewable generation and load predictions [6]-[10].

The scalability of centralised energy management is of concern, since renewable generation and load predictions are required for all of the prosumers [11]. Sending all of this information to a central processor introduces privacy and security issues [12]. To address this, distributed energy management strategies have been proposed, including dual decomposition [13], [14], alternating direction method of multipliers (ADMM) [15], [16] and distributed consensus based algorithms [17], [18]. Distributed optimisation methods based on dual prices (e.g. dual decomposition, ADMM) can be interpreted as competitive market mechanisms [19].

Existing energy management strategies treat energy as a homogeneous product. However, it has been found that prosumers are willing to buy/sell energy at different prices depending on its source/destination. Studies in several countries have found that consumers are willing to pay a premium for renewable energy [20]-[23]. A choice modelling experiment undertaken in Germany found a greater willingness to pay for local generation, and for certain renewable technologies over others [24]. In [25], latent class modelling was used to group residential consumers in Denmark according to their energy preferences. Three distinct groups were identified, 'value seeking consumers', 'price sensitive consumers' and 'green consumers'. The prosumer survey in [26] found a majority of respondents would be willing to share unused energy resources with their local community.

Peer-to-peer (P2P) market platforms have emerged in a range of sectors, allowing small suppliers to compete with traditional providers of goods and services [27], [28]. Prominent examples include Uber for $\mathrm{P} 2 \mathrm{P}$ transport, Airbnb for $\mathrm{P} 2 \mathrm{P}$ accommodation and eBay for $\mathrm{P} 2 \mathrm{P}$ auctions. $\mathrm{P} 2 \mathrm{P}$ platforms allow for matching between suppliers and consumers with a high degree of heterogeneity [29]. With the rise of distributed renewable sources, energy storage and communications at the distribution level, P2P energy market platforms could be used to coordinate energy trading between prosumers [30], [31]. In addition, a P2P energy market platform offers the new op- 


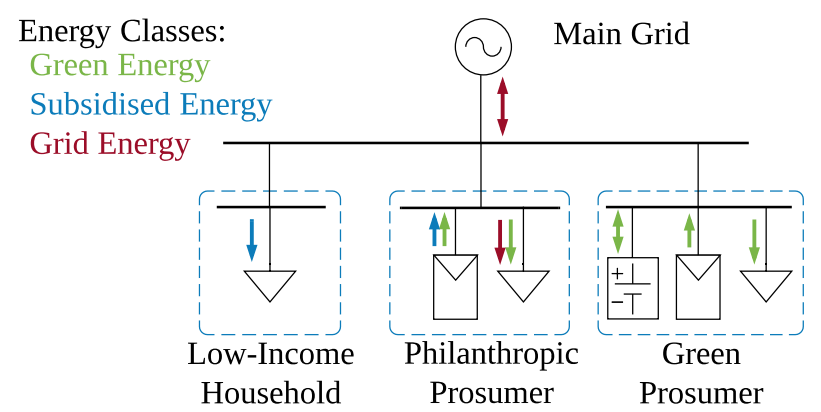

Fig. 1. A distribution network with three prosumers and three energy classes. The green prosumer is willing to pay a premium for green energy. The philanthropic prosumer is willing to supply subsidised energy to the lowincome household at a reduced price.

portunity to incorporate the heterogeneous energy preferences of individual prosumers into the management of the network [32].

This paper proposes a $\mathrm{P} 2 \mathrm{P}$ energy market platform based on the new concept of multi-class energy management, to coordinate trading between prosumers with heterogeneous (i.e. beyond purely financial) preferences. The proposed P2P energy market platform has the following key features:

(i) The new concept of 'energy classes' allows energy to be treated as a heterogeneous product, based on attributes of its source which are perceived by prosumers to have value. Examples include generation technology, location in the network and the owner's reputation.

(ii) The P2P platform minimises costs associated with power losses and battery depreciation, while providing added value by accounting for the prosumers' individual preferences for the source/destination of the energy they consume/produce.

(iii) The decomposable structure of the multi-class energy management problem is exploited to devise a distributed price-directed optimisation mechanism, using ADMM. This provides scalability and prosumer data privacy.

(iv) Receding horizon model predictive control (MPC) allows the prosumers to adjust their planned power flows based on differences between the actual and predicted wholesale energy price, their renewable generation and their loads.

The operation of the proposed $\mathrm{P} 2 \mathrm{P}$ energy market platform is verified for the IEEE European Low Voltage Test Feeder, with 55 subscribed prosumers.

The rest of this paper is organised as follows. Section II presents the principle of operation of the proposed $\mathrm{P} 2 \mathrm{P}$ energy market platform based on multi-class energy management. In Section III, the centralised multi-class energy management problem is formulated. In Section IV, ADMM is used to devise the distributed price-directed optimisation mechanism. Section $\mathrm{V}$ presents results verifying the operation of the proposed $\mathrm{P} 2 \mathrm{P}$ energy market platform. Section VI concludes the paper.

\section{PRINCIPLE OF OPERATION}

This study considers energy trading between distribution network prosumers, each with local loads, renewable generation sources and/or battery energy storage systems. A P2P

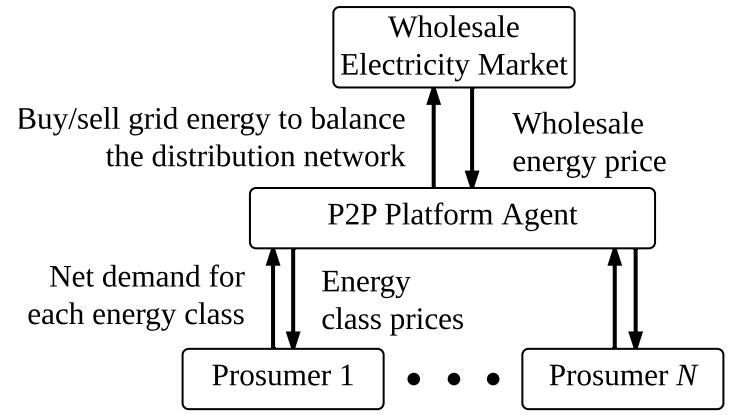

Fig. 2. A block diagram of the proposed P2P energy market platform.

energy market platform is proposed to allow the prosumers to trade energy with each other, and with the main grid through the wholesale electricity market.

The P2P energy market platform has the following objectives:

1) Provide social welfare maximising power flows between the prosumers. The solution needs to account for the prosumers' heterogeneous energy supply/demand preferences, battery depreciation costs and the cost of buying energy from the wholesale electricity market.

2) Satisfy power network constraints and prosumer energy resource constraints. The distribution network power balance must be maintained, and each prosumer has renewable generation, energy storage and load constraints.

3) Satisfy informational constraints for scalability and data privacy. Only the $\mathrm{P} 2 \mathrm{P}$ platform has access to the wholesale electricity market and each prosumers' energy resource capacities, preferences and renewable generation and load predictions are private information.

It is assumed the prosumers have individual energy preferences. For example:

- Prosumers may prefer to obtain the best financial return, regardless of the source/destination of their energy.

- Prosumers may prefer to obtain energy from local sources or particular generation technologies.

- Prosumers may prefer to trade energy with particular subscribers (e.g. friends/relatives, low-income residents, community organisations).

To account for the prosumers' heterogeneous preferences, each unit of energy in the distribution network is assigned an 'energy class' relating to relevant attributes of its source. The prosumer preferences are incorporated into the proposed $\mathrm{P} 2 \mathrm{P}$ market platform through utility functions (envisaged as being set by users), giving their additional willingness to pay to obtain, or to supply, energy with these attributes.

Prosumers wishing only to obtain the best financial return would set their utility functions for each class of energy to zero. The proposed $\mathrm{P} 2 \mathrm{P}$ market platform still provides value to these prosumers, by allowing them to trade energy with the wholesale market and other prosumers to best utilise the capacity of their energy resources.

Fig. 1 shows an example distribution network where multiclass energy management adds value. The distribution network 
has a 'green prosumer', a 'philanthropic prosumer' and a 'low-income household'. Three energy classes are introduced to account for their preferences; 'green energy', 'subsidised energy' and 'grid energy'.

A platform agent is introduced to act as an auctioneer and to allow energy trading between the prosumers and the wholesale electricity market. The platform agent sets the price of each energy class in the distribution network. A block diagram of the proposed P2P energy market platform is shown in Fig. 2.

Distributed price-directed optimisation parallelises the multi-class energy management problem. At each wholesale market trading interval, the prosumers solve local optimisation sub-problems to schedule their renewable sources and battery energy storage systems, considering their generation and load predictions and the price of each class of energy. The platform agent adjusts the energy class prices, considering the prosumer energy demands, the wholesale energy price, wholesale price predictions and expected losses. Iteratively, the prosumers and platform agent reach agreement on a schedule of social welfare maximising power flows.

Receding horizon MPC is used to provide a real-time implementation. Once the energy class prices have converged for the current trading interval, the trading interval recedes by a step and the prosumers update their local battery SoC estimates, generation predictions and load predictions.

It is assumed that the $\mathrm{P} 2 \mathrm{P}$ energy market platform operates as a price-taker in the wholesale electricity market. The platform agent is programmed to set prices that maximise social welfare, and the individual prosumers are programmed to operate as competitive price-takers. This is desirable for all participants if the platform owner faces competition from other potential platforms, so it cannot set prices to maximise profits, and there are sufficient prosumers so that they cannot improve their position through strategic price-setting behaviour [33]. If these conditions are not met over the long term, the platform owner/prosumers with market power may need to be compensated to incentivise their participation.

The proposed $\mathrm{P} 2 \mathrm{P}$ energy market platform allows smallscale prosumers to trade energy with one another, and with the wholesale market, rather than relying on a traditional retail supplier. Two distinct types of P2P markets can be identified [29]: 1) P2P markets with individual sellers responsible for adjusting prices (e.g. eBay and Airbnb), and 2) P2P markets with a centralised price setting mechanism to balance supply and demand (e.g. Uber). The proposed P2P market platform is a member of the second type. It should be noted that the platform's description as a $\mathrm{P} 2 \mathrm{P}$ market is separate from its underlying network architecture, which is distributed, but not fully P2P [34].

The proposed $\mathrm{P} 2 \mathrm{P}$ energy market platform operates in a distribution network so that local prosumer energy balancing can be incentivised, and the costs associated with importing energy from the main grid can be accounted for. Through the $\mathrm{P} 2 \mathrm{P}$ market, prosumers can trade energy with one another and the wholesale market without a retail supplier. However, retail suppliers could still have an important role, providing services such as invoicing, real-time metering and local energy management [35]. Also, small-scale prosumers may not wish to

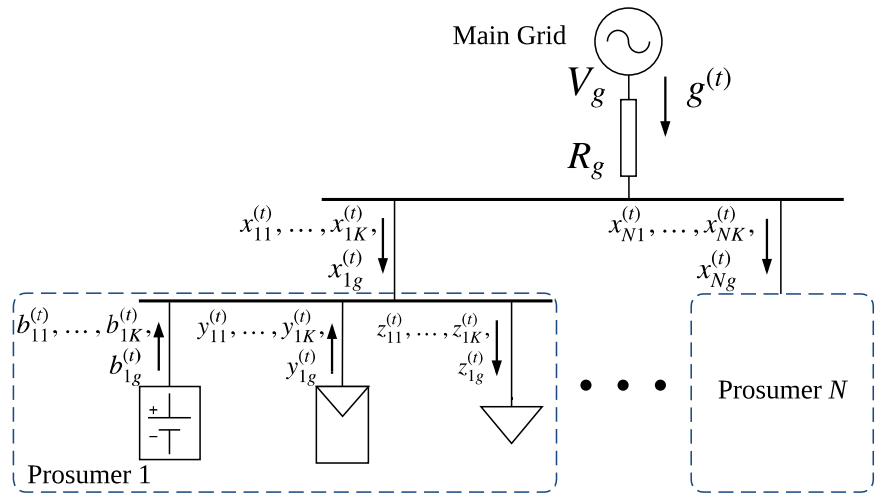

Fig. 3. The simplified real power distribution network model used to formulate the multi-class energy management problem. Each prosumer has a battery energy storage system, renewable source and/or load.

be exposed to fluctuating wholesale energy prices. Prosumers could contract with retail suppliers to obtain predictable energy prices, and then their supplier could act on their behalf within the P2P platform based on their energy preferences.

\section{Multi-Class Energy Management}

Let the set of distribution network prosumers be $\mathcal{N}=$ $\{1, \ldots, N\}$ and the set of energy classes generated by the prosumers be $\mathcal{K}=\{1, \ldots, K\}$. The MPC time horizon is $\mathcal{T}=\left\{t_{0}, \ldots, T\right\}$, where $t_{0}$ is the current trading interval. Each time interval $t \in \mathcal{T}$ has its own duration $T_{s}^{(t)}$.

Wholesale electricity markets operate with high temporal resolution trading intervals (e.g. 5 minute intervals for the Australian National Electricity Market (NEM)), while price predictions available from day-ahead trading and day-ahead prosumer renewable generation and load predictions often have lower temporal resolution (e.g. 2 hour intervals). Therefore, each time horizon interval may have a different duration. For example, with a 5 minute trading interval and 2 hour dayahead predictions, $t_{0}$ lasts for 5 minutes, $t_{0}+1$ lasts from the end of interval $t_{0}$ until the next full 2 hour interval and $\left\{t_{0}+2, \ldots, T\right\}$ are the remaining full 2 hour intervals until the end of the day.

For prosumer $i \in \mathcal{N}$, time interval $t \in \mathcal{T}$ and energy class $k \in \mathcal{K} ; y_{i k}^{(t)}$ is the average renewable source output power, $b_{i k}^{(t)}$ is the average battery output power, $z_{i k}^{(t)}$ is the average allocated load power and $x_{i k}^{(t)}$ is the average net power supplied to the prosumer. For grid class energy, the renewable generation export, average battery output power, allocated load power and net supplied power are $y_{i g}^{(t)}, b_{i g}^{(t)}, z_{i g}^{(t)}$ and $x_{i g}^{(t)}$.

Implementing the proposed $\mathrm{P} 2 \mathrm{P}$ energy market platform in real-time requires computational speed. In recognition of this, simplifying assumptions are made to obtain a convex formulation of the proposed multi-class energy management problem, since fast and robust solvers are readily available for convex optimisation problems [36]. Energy management strategies often use simplified models to obtain a convex formulation [6]-[8], [13]-[17]. In Section IV, the convex formulation allows the proposed multi-class energy management problem to be parallelised using distributed price-directed optimisation. 
Fig. 3 shows the simplified real power distribution network model used to obtain a convex formulation for the proposed multi-class energy management problem. $g^{(t)}$ is the average grid power supplied to the distribution network during interval $t, V_{g}$ is the nominal RMS grid voltage and $R_{g}$ is the average resistance between the main grid and the distribution network prosumers. Losses between the main grid and the distribution network are approximated by $\frac{R_{g}}{V_{g}^{2}} g^{(t) 2}$, based on the assumption of small network voltage drops and relatively low reactive power flows [37]. Losses due to power flows between prosumers are assumed to be relatively small and are not included in the model.

The platform agent's cost function is given by,

$$
f_{g}=\sum_{t \in \mathcal{T}} f_{g}^{(t)}=\sum_{t \in \mathcal{T}} C_{g}^{(t)} T_{s}^{(t)}\left(g^{(t)}+\frac{R_{g}}{V_{g}^{2}} g^{(t) 2}\right)
$$

$C_{g}^{(t)}$ is the predicted wholesale energy price at interval $t$.

The cost functions for the prosumers, $i \in \mathcal{N}$, are given by,

$f_{i}=\sum_{t \in \mathcal{T}} T_{s}^{(t)}\left[C_{d e p}\left(B_{c i}^{(t)}+B_{d i}^{(t)}\right)-\sum_{k \in \mathcal{K}}\left(u_{i k}^{d} z_{i k}^{(t)}+u_{i k}^{s} y_{i k}^{(t)}\right)\right]$.

$B_{c i}^{(t)} \geq 0$ and $B_{d i}^{(t)} \geq 0$ are the total battery charging and discharging powers. $C_{d e p}$ is the cost of battery depreciation, assuming a uniform energy throughput degradation rate [38]. Prosumer $i$ 's preference for its demand to be satisfied by energy of class $k$ is modelled by the utility coefficient, $u_{i k}^{d} \geq 0$. Prosumer $i$ 's preference for supplying energy of class $k$, rather than selling to the grid, is modelled by the utility coefficient, $u_{i k}^{s} \geq 0$.

For each interval of the time horizon, $t \in \mathcal{T}$, the following power flow constraints are required to ensure the power flows of each energy class are balanced.

$$
\begin{aligned}
& \sum_{i \in \mathcal{N}} x_{i g}^{(t)}-g^{(t)}=0 . \\
& \sum_{i \in \mathcal{N}} x_{i k}^{(t)}=0, \forall k \in \mathcal{K} .
\end{aligned}
$$

For each prosumer, $i \in \mathcal{N}$, the local power flows must match the net supplied power of each energy class, introducing the following coupling constraints,

$$
\begin{aligned}
& z_{i g}^{(t)}-x_{i g}^{(t)}-y_{i g}^{(t)}-b_{i g}^{(t)}=0, \forall t \in \mathcal{T} . \\
& z_{i k}^{(t)}-x_{i k}^{(t)}-y_{i k}^{(t)}-b_{i k}^{(t)}=0, \forall k \in \mathcal{K}, \forall t \in \mathcal{T} .
\end{aligned}
$$

Also, each prosumer, $i \in \mathcal{N}$, has load constraints, renewable generation constraints and battery constraints. The prosumer load constraints require that the total power allocated to its load meets its load predictions, $z_{i}^{\text {pred(t) }}, \forall t \in \mathcal{T}$. Let $\mathcal{K}_{i} \subseteq \mathcal{K}$ be the set of energy classes that prosumer $i$ is allowed to use to supply its loads (e.g. in the example from Fig. 1, subsidised energy is reserved for low-income households).

$$
\begin{aligned}
& \sum_{k \in \mathcal{K}_{i}} z_{i k}^{(t)}+z_{i g}^{(t)}=z_{i}^{\text {pred }(t)}, \forall t \in \mathcal{T} . \\
& 0 \leq z_{i g}^{(t)}, 0 \leq z_{i k}^{(t)}, \forall k \in \mathcal{K}, \forall t \in \mathcal{T} .
\end{aligned}
$$

For prosumer $i \in \mathcal{N}$, let $y_{i}^{p r e d(t)}$ be the predicted generation capacity at interval $t$.

$$
\begin{aligned}
& \sum_{k \in \mathcal{K}} y_{i k}^{(t)}+y_{i g}=y_{i}^{p r e d(t)}, \forall t \in \mathcal{T} . \\
& 0 \leq y_{i g}^{(t)}, \quad 0 \leq y_{i k}^{(t)}, \forall k \in \mathcal{K}, \forall t \in \mathcal{T} .
\end{aligned}
$$

For prosumer $i \in \mathcal{N}$, let $s_{i g}^{(t)}, s_{i k}^{(t)}, k \in \mathcal{K}$ be the battery SoC allocated to each energy class and let $E_{b i}$ be the battery energy capacity. A linear SoC model is used to develop constraints to keep the total battery SoC between $s_{i}^{\text {min }}$ and $s_{i}^{\max }$ over the time horizon, and to enforce a required final SoC level of $s_{i}^{(T+1)}$. Prosumer $i$ 's SoC constraints are given by,

$$
\begin{gathered}
s_{i}^{\text {min }} \mathbf{1} \leq \sum_{k \in \mathcal{K}}\left(s_{i k}^{\left(t_{0}\right)} \mathbf{1}+A_{b i} \mathbf{b}_{i k}\right)+s_{i g}^{\left(t_{0}\right)} \mathbf{1}+A_{b i} \mathbf{b}_{i g} \leq s_{i}^{\max } \mathbf{1}, \\
\sum_{k \in \mathcal{K}}\left(s_{i k}^{\left(t_{0}\right)}+a_{b i}^{(T)} \mathbf{b}_{i k}\right)+s_{i g}^{\left(t_{0}\right)}+a_{b i}^{(T)} \mathbf{b}_{i g}=s_{i}^{(T+1)}, \\
\mathbf{b}_{i k}=\left[\begin{array}{lll}
b_{i k}^{\left(t_{0}\right)} & \cdots & b_{i k}^{(T)}
\end{array}\right]^{\top}, \mathbf{b}_{i g}=\left[\begin{array}{lll}
b_{i g}^{\left(t_{0}\right)} & \cdots & b_{i g}^{(T)}
\end{array}\right]^{\top}, \\
A_{b i}=\left[\begin{array}{c}
a_{b i}^{\left(t_{0}\right)} \\
a_{b i}^{\left(t_{0}+1\right)} \\
\vdots \\
a_{b i}^{(T)}
\end{array}\right]=-\frac{1}{E_{b i}}\left[\begin{array}{cccc}
T_{s}^{\left(t_{0}\right)} & 0 & \cdots & 0 \\
T_{s}^{\left(t_{0}\right)} & T_{s}^{\left(t_{0}+1\right)} & \ddots & \vdots \\
\vdots & \vdots & \ddots & 0 \\
T_{s}^{\left(t_{0}\right)} & T_{s}^{\left(t_{0}+1\right)} & \cdots & T_{s}^{(T)}
\end{array}\right] .
\end{gathered}
$$

$\mathbf{1}$ is a vector with $|\mathcal{T}|$ elements, all equal to 1 .

The following additional constraints are needed since negative SoC levels are not allowed.

$$
\begin{aligned}
& \mathbf{0} \leq s_{i k}^{\left(t_{0}\right)} \mathbf{1}+A_{b i}\left(\mathbf{b}_{i k}+\mathbf{b}_{i k g}\right), \forall k \in \mathcal{K}, \forall t \in \mathcal{T} \\
& \mathbf{0} \leq s_{i g}^{\left(t_{0}\right)} \mathbf{1}+A_{b i}\left(\mathbf{b}_{i g}-\sum_{k \in \mathcal{K}} \mathbf{b}_{i k g}\right), \forall t \in \mathcal{T} .
\end{aligned}
$$

$\mathbf{0}$ is a vector with $|\mathcal{T}|$ elements, all equal to 0 . In (13), (14), $\mathbf{b}_{i k g}=\left[\begin{array}{lll}b_{i k g}^{\left(t_{0}\right)} & \cdots & b_{i k g}^{(T)}\end{array}\right]^{\top}$, where $b_{i k g}^{(t)}, t \in \mathcal{T}$ is the amount of class $k$ energy stored as grid energy. Certain energy classes, $k \in \mathcal{K}_{g} \subseteq \mathcal{K}$, are allowed to be substituted for and stored as grid energy (e.g. in the example from Fig. 1, green energy is traded at a premium and therefore may be substituted for grid energy). The following constraints are introduced to restrict the energy classes that can be substituted and stored as grid energy.

$$
\begin{aligned}
& 0 \leq b_{i k g}^{(t)}, \forall k \in \mathcal{K}_{g}, \forall t \in \mathcal{T}, \\
& b_{i k g}^{(t)}=0, \forall k \in \mathcal{K} \backslash \mathcal{K}_{g}, \quad \forall t \in \mathcal{T} .
\end{aligned}
$$

The battery also has output power limits. Let $B_{c i}^{\max }$ be the maximum charging power and $B_{d i}^{\max }$ be the maximum discharging power. The battery output power constraints for prosumer $i$ are given by,

$$
-B_{c i}^{\max } \leq \sum_{k \in \mathcal{K}} b_{i k}^{(t)}+b_{i g}^{(t)} \leq B_{d i}^{\max }, \forall t \in \mathcal{T}
$$

The following additional constraints link the total battery charging power $B_{c i}^{(t)}$ and discharging power $B_{d i}^{(t)}$ at each time 
interval to the battery output powers of each energy class,

$$
\begin{aligned}
& B_{d i}^{(t)}-B_{c i}^{(t)}=\sum_{k \in \mathcal{K}} b_{i k}^{(t)}+b_{i g}^{(t)}, \forall t \in \mathcal{T}, \\
& 0 \leq B_{c i}^{(t)}, 0 \leq B_{d i}^{(t)}, \forall t \in \mathcal{T} .
\end{aligned}
$$

The battery depreciation model in (2) requires that for each interval $t \in \mathcal{T}$, either $B_{d i}^{(t)}=0$, or $B_{c i}^{(t)}=0$. This will be the case, since for any solution with both $B_{d i}^{(t)}$ and $B_{c i}^{(t)}$ greater than zero, there is a lower cost feasible solution with one equal to zero, and the other at a smaller value [9].

The following decision variable vectors are defined for the prosumers $i \in \mathcal{N}$,

$\mathbf{x}_{i}=\left(x_{i g}^{(t)}, x_{i k}^{(t)} \mid k \in \mathcal{K}, t \in \mathcal{T}\right), \mathbf{y}_{i}=\left(y_{i g}^{(t)}, y_{i k}^{(t)} \mid k \in \mathcal{K}, t \in \mathcal{T}\right)$, $\mathbf{z}_{i}=\left(z_{i g}^{(t)}, z_{i k}^{(t)} \mid k \in \mathcal{K}, t \in \mathcal{T}\right)$,

$\mathbf{b}_{i}=\left(b_{i g}^{(t)}, b_{i k}^{(t)}, b_{i k g}^{(t)}, B_{c i}^{(t)}, B_{d i}^{(t)} \mid k \in \mathcal{K}, t \in \mathcal{T}\right)$,

The centralised formulation of the proposed multi-class energy management problem can be stated as,

$$
\begin{aligned}
& \underset{\left(\mathbf{x}_{i}, \mathbf{y}_{i}, \mathbf{z}_{i}, \mathbf{b}_{i} \mid i \in \mathcal{N}\right),}{\operatorname{minimise}} \quad f=f_{g}+\sum_{i \in \mathcal{N}} f_{i} \\
& \left(g^{(t)} \mid t \in \mathcal{T}\right) \\
& \text { subject to } \\
& \text { (3), (4), } \forall t \in \mathcal{T}, \quad \text { (Power Flow Const.) } \\
& \text { (5), (6), } \forall i \in \mathcal{N} \text {. (Coupling Const.) } \\
& \text { (7)-(19), } \forall i \in \mathcal{N} \text {. (Prosumer Const.) }
\end{aligned}
$$

Since (20) is a convex quadratic program with affine constraints, strong duality holds assuming there is a feasible solution [36].

Receding horizon MPC can be used to implement multiclass energy management for real-time control of the distribution network prosumers. During each wholesale market trading interval:

1) For the current trading interval, $t_{0}$, the current wholesale electricity price and updated price predictions, $C_{g}^{(t)}, \forall t \in$ $\mathcal{T}$, are obtained. Also, each prosumer, $i \in \mathcal{N}$, estimates its current SoC allocated to each energy class, $s_{i g}^{\left(t_{0}\right)}, s_{i k}^{\left(t_{0}\right)}, \forall k \in \mathcal{K}$, and obtains updated renewable generation and load predictions, $y_{i}^{\operatorname{pred}(t)}, z_{i}^{\operatorname{pred}(t)}, \forall t \in \mathcal{T}$.

2) The multi-class energy management problem (20) is solved.

3) Each prosumer, $i \in \mathcal{N}$, implements the optimal renewable output powers, $y_{i g}^{*\left(t_{0}\right)}, y_{i k}^{*\left(t_{0}\right)}, \forall k \in \mathcal{K}$, and battery output powers, $b_{i g}^{*\left(t_{0}\right)}, b_{i k}^{*\left(t_{0}\right)}, b_{i k g}^{*\left(t_{0}\right)}, \forall k \in \mathcal{K}$, obtained for the current trading interval, $t_{0}$, by using them as lower level controller references for their energy sources.

4) The MPC time horizon recedes by a step, $t_{0} \leftarrow t_{0}+1$, for the next trading interval.

To obtain a convex problem formulation, the utility prosumers receive for additional energy in (2) must be concave. In this study, it is assumed the prosumers receive constant marginal utility for using their preferred energy classes. However, any function with constant or diminishing marginal returns for additional energy could be used. For example, prosumers with smart thermostats could be modelled as receiving high marginal utility for energy used to reach a minimum desired temperature level, and diminishing marginal utility for additional energy usage [39], [40].

\section{Distributed Price-Directed Optimis ATION}

To satisfy informational constraints for scalability and data privacy, the decomposable structure of the multi-class energy management problem is exploited to devise a distributed pricedirected optimisation mechanism, using ADMM.

ADMM is a distributed convex optimisation method, which uses iterative local optimisation and dual price adjustments [41]. The dual price adjustments can be interpreted as the tâtonnement process of a Walrasian auction i.e. a competitive market mechanism, where the prices of goods are increased in the case of excess demand and decreased in the case of excess supply, to achieve equilibrium at a set of market clearing prices. ADMM provides convergence even if the local subproblems are constrained [41].

At each wholesale electricity market trading interval, the following distributed mechanism is used to solve the multiclass energy management problem (20).

The platform agent initialises the net power of each energy class assigned to the prosumers over the time horizon, $x_{i g}^{(t)}(0), x_{i k}^{(t)}(0), k \in \mathcal{K}, t \in \mathcal{T}$, as well as dual price variables, $\nu_{g}^{(t)}(0), \nu_{k}^{(t)}(0), k \in \mathcal{K}, t \in \mathcal{T}$, which can be interpreted as the prices of each class of energy in the distribution network for each interval of the time horizon. These initial values are sent to the individual prosumers. Then, during each iteration $\kappa$ :

1) In parallel, each prosumer, $i \in \mathcal{N}$, updates its local decision variables for the current iteration, $\kappa$, by minimising an augmented Lagrangian, $L_{i}$, subject to local constraints.

$$
\begin{array}{cl}
\underset{\mathbf{y}_{i}(\kappa), \mathbf{z}_{i}(\kappa), \mathbf{b}_{i}(\kappa)}{\operatorname{minimise}} & L_{i} \\
\text { subject to } & (7)-(19) .
\end{array}
$$

The augmented Lagrangian includes the prosumer cost function, the price of each class of energy over the time horizon and penalty terms for violations of the coupling constraints.

$$
\begin{aligned}
& L_{i}=f_{i}+ \\
& +\sum_{t \in \mathcal{T}}\left[\nu_{g}^{(t)}(\kappa-1)\left(z_{i g}^{(t)}(\kappa)-x_{i g}^{(t)}(\kappa-1)-y_{i g}^{(t)}(\kappa)-b_{i g}^{(t)}(\kappa)\right)\right. \\
& +\frac{\rho}{2}\left(z_{i g}^{(t)}(\kappa)-x_{i g}^{(t)}(\kappa-1)-y_{i g}^{(t)}(\kappa)-b_{i g}^{(t)}(\kappa)\right)^{2} \\
& +\sum_{k \in \mathcal{K}}\left(\nu_{k}^{(t)}(\kappa-1)\left(z_{i k}^{(t)}(\kappa)-x_{i k}^{(t)}(\kappa-1)-y_{i k}^{(t)}(\kappa)-b_{i k}^{(t)}(\kappa)\right)\right. \\
& \left.\left.+\frac{\rho}{2}\left(z_{i k}^{(t)}(\kappa)-x_{i k}^{(t)}(\kappa-1)-y_{i k}^{(t)}(\kappa)-b_{i k}^{(t)}(\kappa)\right)^{2}\right)\right] .
\end{aligned}
$$

$\rho>0$ in the penalty terms is the ADMM step size.

2) The prosumers calculate their net power demands for each energy class over the time horizon, and send this information to the platform agent,

$$
\begin{aligned}
& \tilde{x}_{i g}^{(t)}(\kappa)=z_{i g}^{(t)}(\kappa)-y_{i g}^{(t)}(\kappa)-b_{i g}^{(t)}(\kappa), t \in \mathcal{T}, \\
& \tilde{x}_{i k}^{(t)}(\kappa)=z_{i k}^{(t)}(\kappa)-y_{i k}^{(t)}(\kappa)-b_{i k}^{(t)}(\kappa), k \in \mathcal{K}, t \in \mathcal{T} .
\end{aligned}
$$

3) The platform agent, in parallel for each interval of the time horizon, $t \in \mathcal{T}$, updates the net power of each energy 


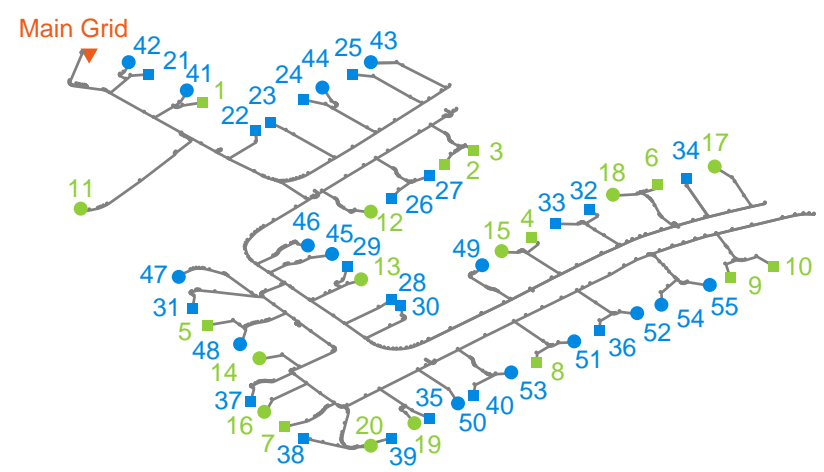

Fig. 4. IEEE European Low Voltage Test Feeder with 55 prosumers. $(\nabla)$ : Main grid point of coupling. ( $/$ ): Green prosumer, (with/without) a PV source and battery. (ם): Philanthropic prosumer, with a PV source and battery. (O): Low-income household.

class supplied to each prosumer and the power imported from the grid, for the current iteration, $\kappa$, by solving,

$$
\underset{\left(g^{(t)}(\kappa), x_{i k}^{(t)}(\kappa), x_{i g}^{(t)}(\kappa) \mid k \in \mathcal{K}, i \in \mathcal{N}\right)}{\operatorname{minimise}} \quad L_{g}^{(t)}
$$

subject to

$$
\text { (3), (4), (Power Flow Const.) }
$$

$L_{g}^{(t)}=f_{g}^{(t)}+\sum_{i \in \mathcal{N}}\left[\nu_{g}^{(t)}(\kappa-1)\left(\tilde{x}_{i g}^{(t)}(\kappa)-x_{i g}^{(t)}(\kappa)\right)\right.$

$+\frac{\rho}{2}\left(\tilde{x}_{i g}^{(t)}(\kappa)-x_{i g}^{(t)}(\kappa)\right)^{2}+\sum_{k \in \mathcal{K}}\left(\nu_{k}^{(t)}(\kappa-1)\left(\tilde{x}_{i k}^{(t)}(\kappa)-x_{i k}^{(t)}(\kappa)\right)\right.$

$\left.\left.+\frac{\rho}{2}\left(\tilde{x}_{i k}^{(t)}(\kappa)-x_{i k}^{(t)}(\kappa)\right)^{2}\right)\right]$.

4) The platform agent then updates the dual prices. For a particular class of energy $k \in \mathcal{K}$ and time interval $t \in \mathcal{T}$, the prosumer coupling constraints (5), (6), will have the same dual price for all $i \in \mathcal{N}$, since relaxations are interchangeable through the power balance constraints (3), (4). This means that only a single dual price is needed for each class of energy at each interval of the time horizon, and the platform agent can update the prices using the coupling constraint mismatch for any prosumer $i \in \mathcal{N}$,

$$
\begin{aligned}
& \left.\nu_{g}^{(t)}(\kappa)=\nu_{g}^{(t)}(\kappa-1)+\rho \tilde{x}_{i g}^{(t)}(\kappa)-x_{i g}^{(t)}(\kappa)\right), t \in \mathcal{T}, \\
& \nu_{k}^{(t)}(\kappa)=\nu_{k}^{(t)}(\kappa-1)+\rho\left(\tilde{x}_{i k}^{(t)}(\kappa)-x_{i k}^{(t)}(\kappa)\right), k \in \mathcal{K}, t \in \mathcal{T} .
\end{aligned}
$$

Since the multi-class energy management problem (20) satisfies strong duality, the decision variables and dual prices will converge to their optimal values [41]. For real-time control, the distributed price-directed optimisation replaces step 2) of the MPC procedure described in Section III.

\section{RESUlts}

The operation of the proposed $\mathrm{P} 2 \mathrm{P}$ energy market platform based on multi-class energy management was verified using the IEEE European Low Voltage Test Feeder [42], with 55 prosumers. The distribution network is shown in Fig. 4. For the distribution network model, a nominal voltage of $V_{g}=415 \mathrm{~V}$ and average resistance between the prosumers and main grid of $R_{g}=0.084 \Omega$ are used.

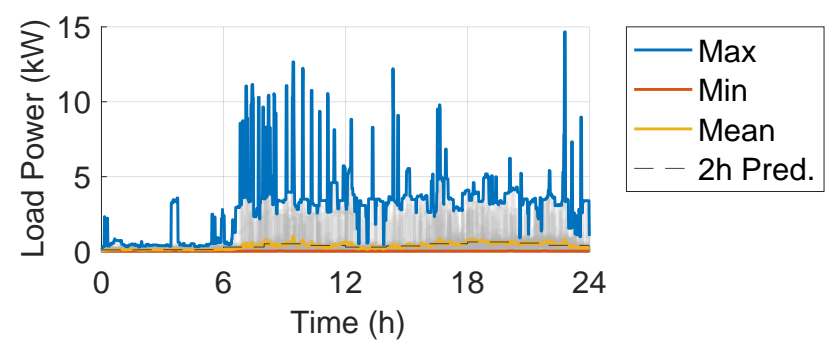

(a) Prosumer load profiles.

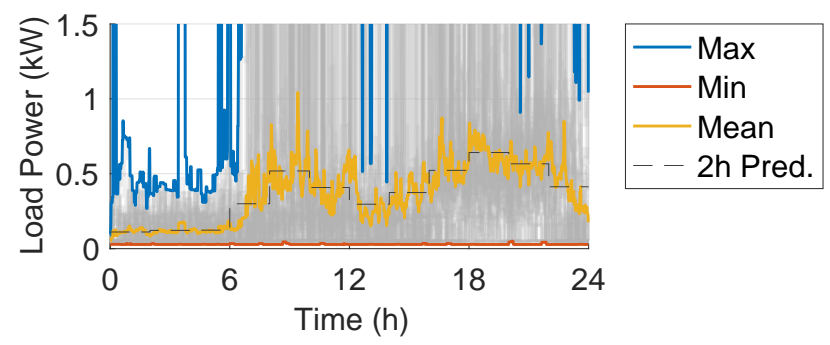

(b) Zoomed view of the prosumer load profiles.

Fig. 5. The grey plots show the individual prosumer load profiles (with $20 \%$ opacity to show overlaps). The maximum, minimum and mean load are also shown, as well as the two hour resolution day-ahead predicted profile used by all of the prosumers.

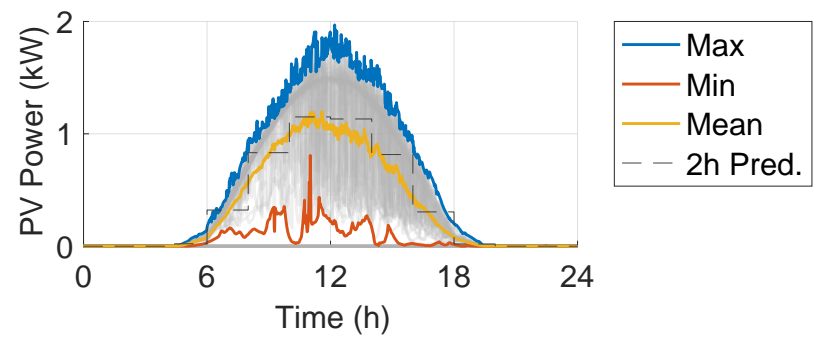

Fig. 6. The grey plots show the individual prosumer PV generation profiles (with $20 \%$ opacity to show overlaps). The maximum, minimum and mean PV generation are also shown, as well as the two hour resolution day-ahead predicted profile used by all of the prosumers with PV sources.

There are three types of prosumers in the distribution network, 'green prosumers', 'philanthropic prosumers' and 'low-income households'. Based on their preferences, three energy classes are introduced; 'grid energy', 'green energy and 'subsidised energy'. Grid energy, is traded with the wholesale market at five minute trading intervals through the platform agent. Green energy is traded at a premium, so it is allowed to be substituted for and stored/sold as grid energy. Only lowincome households are allowed to use subsidised energy.

The green prosumers are labelled 1 to 20 . Of these, 1 to 10 have $1 \mathrm{~kW}$ PV sources and $1 \mathrm{~kW}, 2 \mathrm{kWh}$ battery energy storage systems. The green prosumers obtain additional perceived utility worth $\$ 15 / \mathrm{MWh}$ when their loads are supplied with green energy. The philanthropic prosumers are labelled 21 to 40 , and have $1 \mathrm{~kW}$ PV sources and $1 \mathrm{~kW}, 2 \mathrm{kWh}$ battery energy storage systems. These prosumers obtain additional perceived utility worth $\$ 15 / \mathrm{MWh}$ when they supply subsidised energy to low-income households. The low-income households are labelled 41 to 55 .

The green and philanthropic prosumers' batteries begin at 


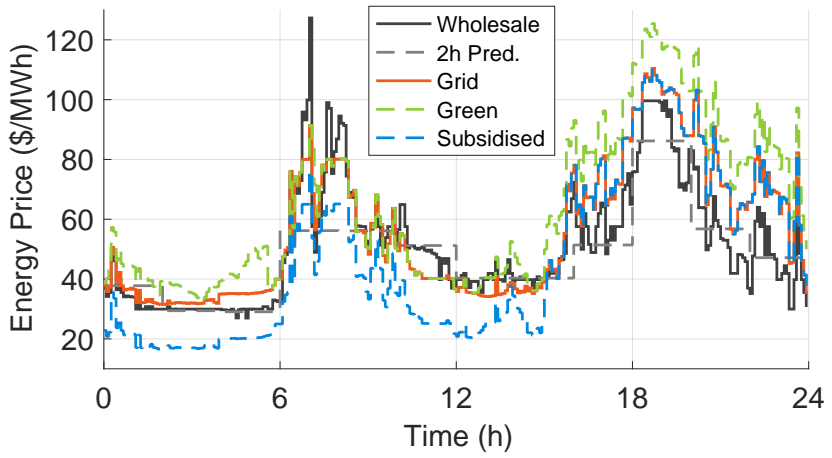

Fig. 7. The wholesale energy market prices and 2 hour resolution predictions, and the prices for green energy, subsidised energy and grid energy set by the platform agent.

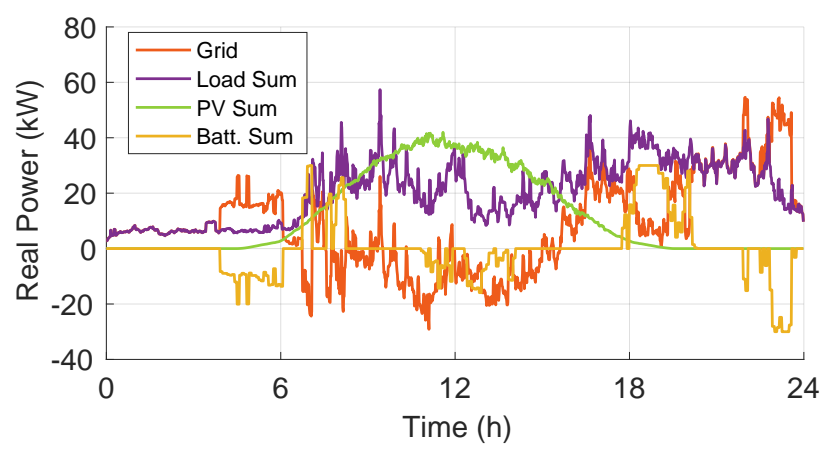

Fig. 8. The total power imported from the grid, and the total prosumer load power, PV generation and battery output power.

$60 \% \mathrm{SoC}$, with green and subsidised energy respectively. The batteries have SoC limits of $20 \%$ to $100 \%$, and a final required SoC of $60 \%$. The cost of battery depreciation is assumed to be $\$ 20 / \mathrm{MWh}$.

One minute resolution load data from the IEEE European Low Voltage Test Feeder is used for the prosumer load profiles. The prosumer load profiles are shown in Fig. 5. The loads have 0.95 power factor and the PV sources and battery energy storage systems have unity power factor.

The prosumer PV generation profiles were generated using temperature and irradiance data with one minute resolution from the NREL Baseline Measurement System (from June 2016, with each day's data used for one of the prosumers) [43]. The PV profiles are shown in Fig. 6.

It is assumed that the prosumers have access to a common set of day-ahead 2 hour resolution load and PV generation predictions, as well as accurate 5 minute ahead predictions of their individual average load and PV generation. The dayahead predicted load profile is based on the average distribution network load. The day-ahead predicted PV generation profile is based on an $80 \%$ rating of the nominal PV generation capacity, which slightly overestimates the actual average PV generation.

Multi-class energy management is implemented using distributed price-directed optimisation and receding horizon MPC. Each five minute wholesale market trading interval, the platform agent sets energy prices based on the current

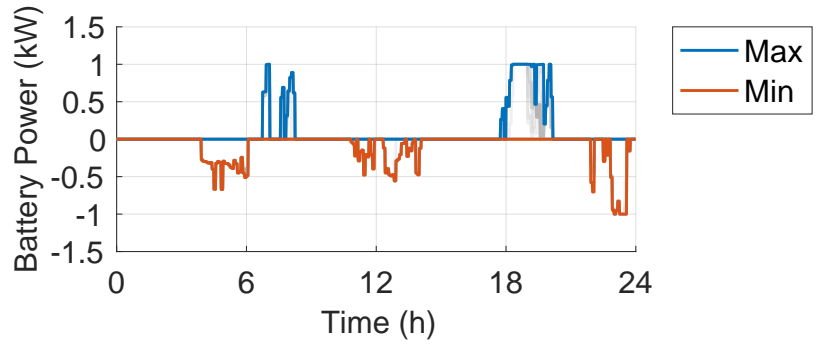

Fig. 9. The output powers of the individual prosumer battery energy storage systems are shown in grey (with $20 \%$ opacity to show overlaps). The maximum and minimum battery output powers are also shown.

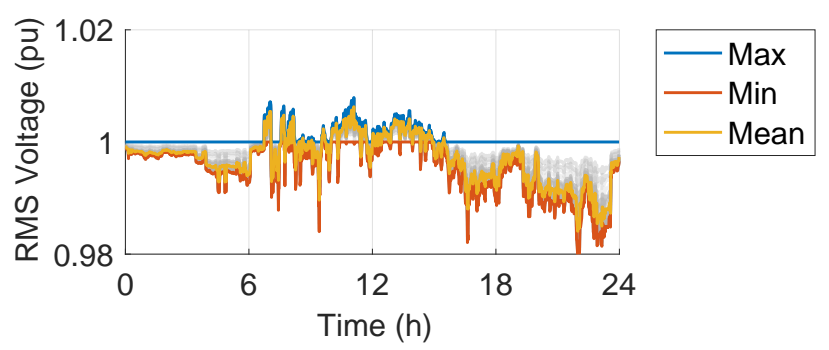

Fig. 10. The voltages of the grid and prosumer buses are shown in grey (with $20 \%$ opacity to show overlaps). The maximum, minimum and mean of the bus voltages are also shown. During the middle of the day the bus voltages rise above the grid voltage ( $1 \mathrm{pu})$, due to PV generation export.

wholesale energy price and 2 hour resolution price predictions. The wholesale energy price and 2 hour resolution day-ahead predictions are shown in Fig. 7, along with the prices for green energy, subsidised energy and grid energy, set by the platform agent during operation. The wholesale energy prices and price predictions (from day-ahead trading) are from the Australian NEM for the 30th of August 2016.

To achieve agreement between the prosumers and the platform agent, the ADMM algorithm is run for 300 iterations at each trading interval, with a step size of $\rho=0.01$. The optimisation sub-problems were solved using IBM's CPLEX solver in MATLAB, on an Intel Core i7-6500U CPU with $8 \mathrm{~GB}$ of RAM. The maximum solution time is $0.0483 \mathrm{~s}$ for the prosumer sub-problems, and $0.0395 \mathrm{~s}$ for the platform agent sub-problems (the average solution time for the centralised multi-class energy management problem is $0.249 \mathrm{~s}$.)

Fig. 8 shows the net power imported from the main grid, and the sum of the prosumer loads, PV generation and battery output powers. The individual battery output powers remain between the charging and discharging limits of $1 \mathrm{~kW}$, as shown in Fig. 9. The resulting bus voltages are shown in Fig. 10.

As shown in Fig. 7, the price of grid energy is above the wholesale energy price when power is imported from the main grid, and below the wholesale energy price when power is exported to the main grid, accounting for expected import/export losses.

Green energy always trades at or above the price of grid energy, since green prosumers are willing to pay a premium to obtain it. Conversely, subsidised energy remains at or below the price of grid energy, since the philanthropic prosumers obtain additional utility for supplying it. 


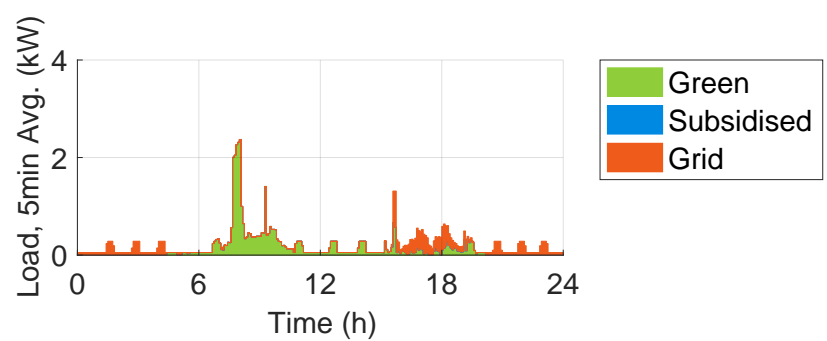

(a) Prosumer 1, a green prosumer with a PV source and battery.

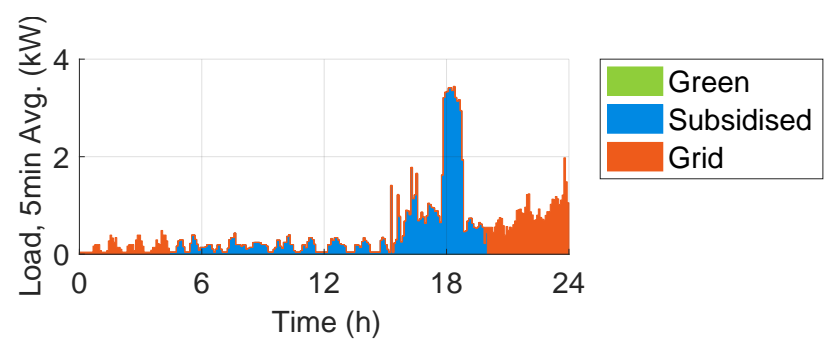

(b) Prosumer 41, a low-income household.

Fig. 11. 5 minute average load profiles for selected prosumers, with energy class allocations.

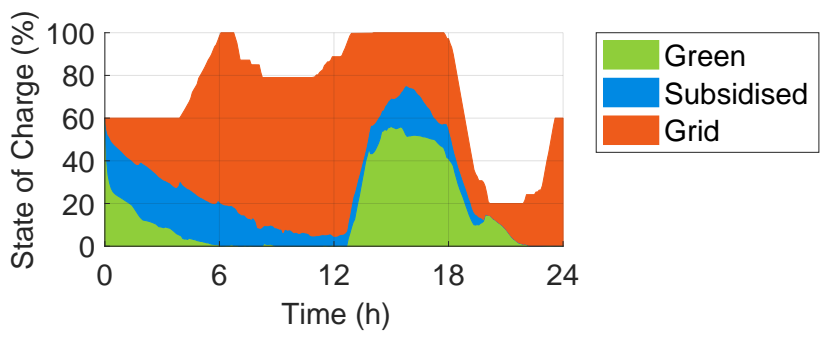

Fig. 12. SoC profile for Prosumer 1's battery, with energy class allocations.

Comparing Fig. 7 and Fig. 9, it can be seen that the prosumer batteries are largely discharged when prices are high, and charged when prices are low.

Fig. 11 shows how selected prosumers allocate the different energy classes to their loads during each 5 minute trading interval. During the middle of the day when there is excess PV generation, Prosumer 1 is able to supply its loads with green energy, which it prefers. During this time, Prosumer 41, a lowincome household, is able to supply its load with subsidised energy. At the start and end of the day, the prosumers use grid energy.

Fig. 12 shows the battery SoC levels of each energy class for Prosumer 1. The battery remains within the SoC limits of $20 \%$ to $100 \%$. At the end of the day, the prosumer buys grid energy to restore its battery to the required $60 \%$ final SoC level.

\section{CONCLUSION}

The new concept of multi-class energy management has been presented, which allows energy to be treated as a heterogeneous product, based on attributes of its source that are perceived by prosumers to have value. This has been used for a P2P energy market platform which coordinates energy trading between distribution network prosumers and the wholesale electricity market. The P2P energy market platform adds value by accounting for individual prosumer energy preferences, which could be financial, social, philanthropic or environmental. The multi-class energy management problem has been parallelised using distributed price-directed optimisation, providing scalability and prosumer data privacy. Receding horizon MPC has been used for a real-time implementation.

There are several important areas for future research. First, it has been assumed that the $\mathrm{P} 2 \mathrm{P}$ energy market platform is a relatively small participant in the wholesale market, and does not have a significant effect on wholesale energy prices. However, if prosumer energy trading becomes widespread, this assumption may no longer be valid. P2P energy market platforms will need to be carefully designed to ensure feedback effects between transmission and distribution system operations do not reduce system stability. One approach could be to introduce an additional penalty term into the platform agent's cost function (1) to limit large imports/exports of power. The aim would be to incentivise the use of local energy storage for smoothing spikes in local generation and demand, reducing the need for balancing at the transmission system level.

Another important area for future research could be the design of new mechanisms allowing the P2P platform operator and prosumers to agree on a limited common set of energy classes which provide them with the most value.

Finally, this study has assumed the platform owner and prosumers face significant competition, and therefore cannot improve their position through strategic price-setting behaviour. If the platform owner does not face external competition, it may be more appropriate to model its interactions with the prosumers using a bi-level optimisation approach, with the platform owner setting prices to maximise its own profits [44]. If individual prosumers have market power, they may need to be incentivised to participate in the $\mathrm{P} 2 \mathrm{P}$ energy market platform. A promising area for future research could be the use of tools from cooperative game theory (e.g. [45], [46]) to design allocation mechanisms which compensate for this by dividing the additional profits obtained when prosumers schedule their energy resources cooperatively rather than individually.

\section{REFERENCES}

[1] A. Dimeas, S. Drenkard, N. Hatziargyriou, S. Karnouskos, K. Kok, J. Ringelstein, and A. Weidlich, "Smart Houses in the Smart Grid: Developing an interactive network." IEEE Electrification Magazine, vol. 2, no. 1, pp. 81-93, Mar. 2014.

[2] R. Wilson, "Architecture of Power Markets," Econometrica, vol. 70, no. 4, pp. 1299-1340, 2002.

[3] X. Yu and Y. Xue, "Smart Grids : A Cyber - Physical Systems Perspective," Proceedings of the IEEE, vol. 104, no. 5, May 2016.

[4] A. Mohd, E. Ortjohann, A. Schmelter, N. Hamsic, and D. Morton, "Challenges in integrating distributed energy storage systems into future smart grid," in 2008 IEEE International Symposium on Industrial Electronics, June 2008, pp. 1627-1632.

[5] Kai Ma, Guoqiang Hu, and C. J. Spanos, "Distributed Energy Consumption Control via Real-Time Pricing Feedback in Smart Grid," IEEE Trans. Control Syst. Technol., vol. 22, no. 5, pp. 1907-1914, Sept. 2014.

[6] E. Mayhorn, K. Kalsi, M. Elizondo, N. Samaan, and K. Butler-Purry, "Optimal control of distributed energy resources using model predictive control," in 2012 IEEE Power and Energy Society General Meeting, July 2012, pp. 1-8.

[7] R. Palma-Behnke, C. Benavides, F. Lanas, B. Severino, L. Reyes, J. Llanos, and D. Saez, "A Microgrid Energy Management System Based on the Rolling Horizon Strategy," IEEE Trans. Smart Grid, vol. 4, no. 2, pp. 996-1006, jun 2013. 
[8] A. Ouammi, H. Dagdougui, L. Dessaint, and R. Sacile, "Coordinated Model Predictive-Based Power Flows Control in a Cooperative Network of Smart Microgrids," IEEE Trans. Smart Grid, vol. 6, no. 5, pp. 22332244, Sept. 2015.

[9] D. E. Olivares, C. A. Canizares, and M. Kazerani, "A Centralized Energy Management System for Isolated Microgrids," IEEE Trans. Smart Grid, vol. 5, no. 4, pp. 1864-1875, July 2014.

[10] T. Morstyn, B. Hredzak, R. P. Aguilera, and V. G. Agelidis, "Model Predictive Control for Distributed Microgrid Battery Energy Storage Systems," IEEE Trans. Control Syst. Technol., May 2017.

[11] T. Morstyn, B. Hredzak, and V. G. Agelidis, "Control Strategies for Microgrids with Distributed Energy Storage Systems: An Overview," IEEE Trans. Smart Grid, Dec. 2016.

[12] N. Z. Aitzhan and D. Svetinovic, "Security and Privacy in Decentralized Energy Trading through Multi-signatures, Blockchain and Anonymous Messaging Streams," IEEE Trans. Depend. Sec. Comput., vol. PP, Oct. 2016.

[13] H. Dagdougui and R. Sacile, "Decentralized Control of the Power Flows in a Network of Smart Microgrids Modeled as a Team of Cooperative Agents," IEEE Trans. Control Syst. Technol., vol. 22, no. 2, pp. 510-519, Mar. 2014.

[14] A. Cortés and S. Martínez, "On distributed reactive power and storage control on microgrids," International Journal of Robust and Nonlinear Control, Jan. 2016.

[15] M. Kraning, E. Chu, J. Lavaei, and S. Boyd, "Dynamic Network Energy Management via Proximal Message Passing," Foundations and Trends in Optimization, vol. 1, no. 2, pp. 70-122, Nov. 2014.

[16] W. Shi, X. Xie, C.-c. Chu, and R. Gadh, "A distributed optimal energy management strategy for microgrids," in 2014 IEEE International Conference on Smart Grid Communications (SmartGridComm), vol. 6, no. 3, Nov. 2014, pp. 200-205.

[17] G. Hug, S. Kar, and C. Wu, "Consensus+Innovations Approach for Distributed Multiagent Coordination in a Microgrid," IEEE Trans. Smart Grid, vol. 6, no. 4, pp. 1893-1903, July 2015.

[18] T. Morstyn, B. Hredzak, and V. G. Agelidis, "Network Topology Independent Multi-Agent Dynamic Optimal Power Flow for Microgrids with Distributed Energy Storage Systems," IEEE Trans. Smart Grid, Nov. 2016.

[19] M. Karlsson, F. Ygge, and A. Andersson, "Market-Based Approaches to Optimization," Computational Intelligence, vol. 23, no. 1, pp. 92-109, Feb. 2007.

[20] A. Longo, A. Markandya, and M. Petrucci, "The internalization of externalities in the production of electricity: Willingness to pay for the attributes of a policy for renewable energy," Ecological Economics, vol. 67, no. 1, pp. 140-152, Jan. 2008.

[21] J. Zorić and N. Hrovatin, "Household willingness to pay for green electricity in Slovenia," Energy Policy, vol. 47, pp. 180-187, May 2012.

[22] A. Tabi, S. L. Hille, and R. Wüstenhagen, "What makes people seal the green power deal? - Customer segmentation based on choice experiment in Germany," Ecological Economics, vol. 107, pp. 206-215, Sept. 2014.

[23] L. Dagher and H. Harajli, "Willingness to pay for green power in an unreliable electricity sector: Part 1 . The case of the Lebanese residential sector," Renewable and Sustainable Energy Reviews, vol. 50, no. 2015, pp. 1634-1642, May 2015.

[24] J. Kaenzig, S. L. Heinzle, and R. Wüstenhagen, "Whatever the customer wants, the customer gets? Exploring the gap between consumer preferences and default electricity products in Germany," Energy Policy, vol. 53, pp. 311-322, Feb. 2013.

[25] Y. Yang, H. S. Solgaard, and W. Haider, "Value seeking, price sensitive, or green? Analyzing preference heterogeneity among residential energy consumers in Denmark," Energy Research \& Social Science, vol. 6, pp. 15-28, Mar. 2015.

[26] P. G. D. Silva, S. Karnouskos, and D. Ilic, "A survey towards understanding residential prosumers in smart grid neighbourhoods," in Innovative Smart Grid Technologies Europe (ISGT Europe), Oct. 2012, pp. 1-8.

[27] A. Hagiu and J. Wright, "Multi-sided platforms," International Journal of Industrial Organization, vol. 43, pp. 162-174, Nov. 2015.

[28] G. Lougher and S. Kalmanowicz, "EU Competition Law in the Sharing Economy," Journal of European Competition Law \& Practice, vol. 7, no. 2, pp. 87-102, Feb. 2016.

[29] L. Einav, C. Farronato, and J. Levin, "Peer-to-Peer Markets," Annual Review of Economics, vol. 8, no. 1, pp. 615-635, Oct. 2016.

[30] Y. Parag and B. K. Sovacool, "Electricity market design for the prosumer era," Nature Energy, pp. 1-6, Mar. 2016.

[31] T. Morstyn, A. Teytelboym, and M. D. McCulloch, "Bilateral Contract Networks for Peer-to-Peer Energy Trading," IEEE Trans. Smart Grid, 2018.
[32] T. Morstyn, N. Farrell, S. J. Darby, and M. D. McCulloch, "Using peer-to-peer energy-trading platforms to incentivize prosumers to form federated power plants," Nature Energy, vol. 3, no. 2, pp. 94-101, 2018.

[33] P. Thomas, D. Teneketzis, and J. K. Mackie-Mason, "A MarketBased Approach to Optimal Resource Allocation in Integrated-Services Connection-Oriented Networks," Operations Research, vol. 50, no. 4, pp. 603-616, May 2002.

[34] R. Schollmeier, "A definition of peer-to-peer networking for the classification of peer-to-peer architectures and applications," in Proceedings First International Conference on Peer-to-Peer Computing, vol. 15, no. 1, New York, NY, 1985, pp. 101-102.

[35] USEF Foundation, USEF: The Framework Explained, 2015. [Online]. Available: usef.energy

[36] S. Boyd and L. Vandenberghe, Convex Optimization. Cambridge, United Kingdom: Cambridge University Press, 2004.

[37] C. Coffrin, P. Van Hentenryck, and R. Bent, "Approximating line losses and apparent power in AC power flow linearizations," IEEE Power and Energy Society General Meeting, pp. 1-8, 2012.

[38] R. Dufo-López, J. M. Lujano-Rojas, and J. L. Bernal-Agustín, "Comparison of different lead-acid battery lifetime prediction models for use in simulation of stand-alone photovoltaic systems," Applied Energy, vol. 115 , pp. 242-253, Nov. 2013.

[39] Z. Fadlullah, D. M. Quan, and S. Member, "GTES: An Optimized GameTheoretic Demand Side Management Scheme for Smart Grid,' IEEE Syst. J., vol. 8, no. 2, pp. 1-10, 2014.

[40] Y. Liang, D. I. Levine, and Z. J. M. Shen, "Thermostats for the smart grid: Models, benchmarks, and insights," Energy Journal, vol. 33, no. 4, pp. 61-95, 2012.

[41] S. Boyd, N. Parikh, E. Chu, B. Peleato, and J. Eckstein, "Distributed Optimization and Statistical Learning via the Alternating Direction Method of Multipliers," Foundations and Trends in Machine Learning, vol. 3, no. 1, pp. 1-122, 2011.

[42] European Low Voltage Test Feeder, "IEEE PES Distribution Systems Analysis Subcommittee Test Feeders." [Online]. Available: https://ewh.ieee.org/soc/pes/dsacom/testfeeders/

[43] A. Andreas and T. Stoffel, "NREL Solar Radiation Research Laboratory (SRRL): Baseline Measurement System (BMS); Golden, Colorado (Data)," NREL Report No. DA-5500-56488, 1981.

[44] W. Wei, F. Liu, and S. Mei, "Energy Pricing and Dispatch for Smart Grid Retailers Under Demand Response and Market Price Uncertainty," IEEE Trans. Smart Grid, vol. 6, no. 3, pp. 1364-1374, 2015.

[45] E. Baeyens, E. Y. Bitar, P. P. Khargonekar, and K. Poolla, "Coalitional aggregation of wind power," IEEE Trans. Power Syst., vol. 28, no. 4, pp. 3774-3784, 2013.

[46] W. Lee, L. Xiang, R. Schober, and V. W. S. Wong, "Direct electricity trading in smart grid: A coalitional game analysis," IEEE J. Sel. Areas Commun., vol. 32, no. 7, pp. 1398-1411, 2014.

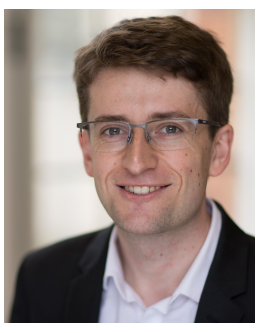

Thomas Morstyn (S'14-M'16) received the B.E. (Hon.) degree from the University of Melbourne, Australia, in 2011, and the PhD degree from the University of New South Wales, Australia, in 2016, both in electrical engineering.

He worked as an electrical engineer in the Rio Tinto Technology and Innovation group between 2012 and 2014. He is currently an Oxford Martin Fellow with the Department of Engineering Science at the University of Oxford. His current research interests include multi-agent control and market design for the integration of distributed energy resources into power systems.

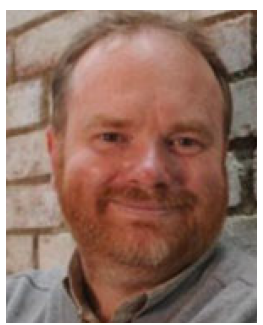

Malcolm D. McCulloch (SM'89) received the B.Sc. (Eng.) and Ph.D. degrees in electrical engineering from the University of the Witwatersrand, Johannesburg, South Africa, in 1986 and 1990, respectively.

In 1993, he joined the University of Oxford, Oxford, U.K., to head up the Energy and Power Group, where he is currently an Associate Professor in the Department of Engineering Science. He is active in the areas of electrical machines, transport, and smart grids. His work addresses transforming existing power networks, designing new power networks for the developing world, developing new technology for electric vehicles, and developing approaches to integrated mobility. 